\title{
Self-Assembly of CdSe Nanoplatelets into Stacks of Controlled Size Induced by Ligand Exchange
}

\author{
Artsiom Antanovich, ${ }^{\dagger}$ Anatol Prudnikau, ${ }^{\dagger}$ Anna Matsukovich, ${ }^{\ddagger}$ Alexander Achtstein, ${ }^{\S}$ \\ and Mikhail Artemyev ${ }^{*} \dagger$ \\ ${ }^{\dagger}$ Research Institute for Physical Chemical Problems, Belarusian State University, Minsk 220030, Belarus \\ ${ }^{\ddagger}$ B. I. Stepanov Institute of Physics, National Academy of Sciences, 220072 Minsk, Belarus \\ ${ }^{\S}$ Institute of Optics and Atomic Physics, Technical University of Berlin, Strasse des 17. Juni 135, 10623 Berlin, Germany
}

Supporting Information

\begin{abstract}
In this paper we present a simple method for the preparation of highly stable colloidal solutions of individual nanoplatelets (NPls) with increased fluorescence quantum yield and a versatile procedure of NPls self-assembly into stacks of controlled size. Dynamic light scattering technique has been demonstrated to be simple and accurate method for in situ studies of the growth kinetics of NPls aggregates. The self-assembly method introduced in this work is based on the exchange of ligands on the surface of CdSe nanoplatelets. Hexadecylphosphonic acid allows control of the average size

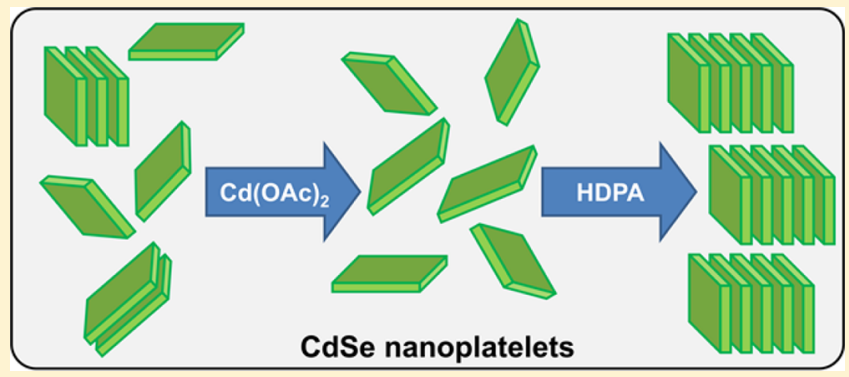
(length) of NPls stacks in a broad range by varying its concentration and reaction time. The main mechanism governing controlled formation of NPls stacks is based on strong van der Waals interaction between rigid brushes of alkyl chains on the surface of neighboring NPls. The interaction strength and, consequently, the length and colloidal stability of stacks have been shown to be dependent on type and concentration of different ligands.
\end{abstract}

\section{INTRODUCTION}

Semiconductor nanoparticles (NPs) are materials of high interest because of their unique properties which make them promising candidates for various applications that range from light-emitting technology to photocatalysis and bioimaging. Most popular NPs are made of $\mathrm{A}^{\mathrm{II}} \mathrm{B}^{\mathrm{VI}}$ semiconductors such as $\mathrm{CdS}, \mathrm{CdSe}$, and $\mathrm{CdTe}$ because their absorption and photoluminescence $(\mathrm{PL})$ bands lie in the visible range. Current synthesis protocols allow preparation of NPs of various forms such as dots, rods, branched particles, and platelets. The latter, commonly called nanoplatelets (NPls) or colloidal quantum wells, were introduced relatively recently. ${ }^{1}$ They have an atomically flat surface, and their thickness is quantized to an integer number of monolayers. ${ }^{2,3}$ As a consequence, if compared to quantum dots (QDs) or nanorods, they exhibit extremely narrow intense bands on both absorption and PL spectra with full width at half maximum (fwhm) as small as 10 $\mathrm{nm}$ and small Stokes shift. To date, various synthesis protocols for the preparation of NPls made of cadmium chalcogenides and various heterostructures on their basis with core-shell ${ }^{4,5}$ and core-wings architecture with type- $\mathrm{I}^{6,7}$ and $-\mathrm{II}^{8-11}$ band alignment were developed. Recent studies have shown that NPls demonstrate optical properties that are superior to those of NPs of other dimensionalities such as giant oscillator strength (GOST), ${ }^{12,13}$ low lasing thresholds, ${ }^{14-16}$ high intrinsic absorption coefficient, ${ }^{17}$ two-photon absorption cross sec- tions, ${ }^{18,19}$ and electrooptical response. ${ }^{20}$ These properties make NPls highly promising materials for light-converters and absorbers, photovoltaic devices, and electro-optical switches, among others.

Although optical properties of semiconductor NPs are mainly determined by the composition, shape, and size of the inorganic core, surface ligands also play an important role; thus, ultrasmall NPs should be in fact considered as complex inorganic-organic composite. Various ligands are used to manipulate solubility of NPs in polar or nonpolar solvents, absorption and PL peak position; intensity, charge trapping, NPs self-assembly, and so on what makes studies of ligandNPs interaction of great importance. ${ }^{21-26}$

At the same time, the composition of the surface ligand layer and self-assembly of $\mathrm{A}^{\mathrm{II}} \mathrm{B}^{\mathrm{VI}} 2 \mathrm{D}$ nanocrystals gained less attention. Recently colloidal assembly of NPls into stacks was achieved by the addition of ethanol that acted as the so-called "bad" solvent. ${ }^{27,28}$ It was shown that in such stacks Förster resonance energy transfer (FRET) occurs up to 50 times faster than Auger recombination that allows implementation of such particles for applications that require high pumping intensity and carrier density, e.g., in optical sensors, light harvesters, and

Received: December 11, 2015

Revised: February 19, 2016

Published: February 19, 2016 

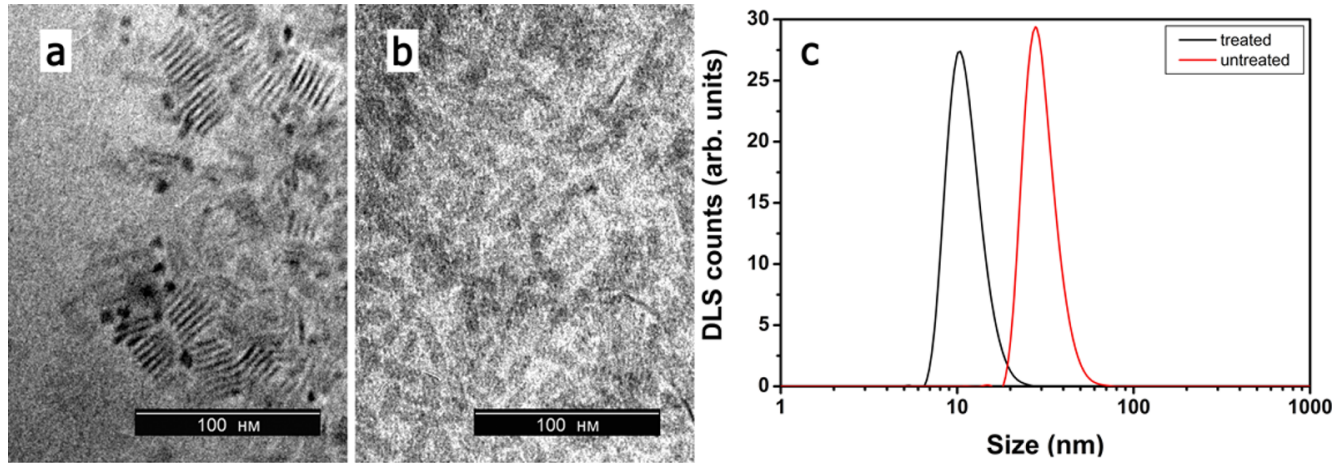

Figure 1. Corresponding TEM images for (a) nontreated and (b) cadmium acetate treated 4.5 ML CdSe NPls. (c) DLS distribution curves for NPls solution in chloroform before (red curve) and after (black curve) the acetate treatment.

LEDs. ${ }^{29}$ In this work we employ another approach to NPls assembly into stacks that is triggered and governed by surface ligand exchange. We also demonstrate that the dynamic light scattering (DLS) technique can be successfully applied for the analysis of NPls aggregation kinetics in situ.

\section{EXPERIMENTAL SECTION}

Materials and Methods. Cadmium acetate dihydrate, zinc acetate dihydrate, cadmium oxide powder, selenium powder (100 mesh), oleic acid, octadecene-1, myristic acid, hexadecanol, and docosane were purchased from Sigma-Aldrich and used as received. $n$-Hexadecylphosphonic acid was purchased from Plasmachem. Stearic acid was purchased from Merck. Chloroform (analytical-reagent-grade) was purchased from Fisher Chemical.

Room-temperature optical absorption spectra were recorded using HR-2000+ spectrometer (Ocean Optics) equipped with Ocean Optics DH-2000 white light source. Photoluminescence measurements were conducted with Jobin-Yvon Fluoromax-2 spectrofluorimeter. TEM images were acquired using LEO 906E electron microscope. DLS measurements were carried out with Malvern Zetasizer Nano ZS90. IR spectra were recorded in the reflectance-absorption mode using Nexus (Thermo Nicolet) spectrometer equipped with PerkinElmer Micro Specular Reflectance Accy-2x plate. PL decay times were measured with fluorimeter PRA System 3000 (Fluorescence Lifetime Instrumentation) by time-correlated photon count method.

Preparation of CdSe Nanoplatelets. CdSe NPls with thicknesses of 4.5 monolayers were synthesized by modified procedure reported previously. ${ }^{8,20}$ Briefly, $35 \mathrm{mg}$ of cadmium oxide, $165 \mathrm{mg}$ of myristic acid, and $15 \mathrm{~mL}$ of octadecene- 1 were introduced into a three-necked flask and heated until the cadmium oxide dissolved completely. After that, the reaction flask was cooled to the room temperature, and $10 \mathrm{mg}$ of selenium powder was added to the as-formed cadmium myristate solution. Then, the reaction flask was degassed, purged with argon, and set to heat to $240{ }^{\circ} \mathrm{C}$. At $193{ }^{\circ} \mathrm{C}, 55 \mathrm{mg}$ of zinc acetate dihydrate powder was swiftly added into the reaction mixture. When the temperature reached $240{ }^{\circ} \mathrm{C}$, the reaction flask was heated for $3 \mathrm{~min}$. After this time, the heating mantle was removed, and at $180{ }^{\circ} \mathrm{C}, 1 \mathrm{~mL}$ of oleic acid was added to the reaction mixture. When reaction mixture cooled down to $80{ }^{\circ} \mathrm{C}$, $\mathrm{CdSe}$ core NPls were precipitated out with isopropanol, isolated by centrifugation at $3000 \mathrm{rpm}$, and redispersed in chloroform. After that, the NPls solution was purified twice by precipitation with acetonitrile and resuspension in chloroform.

Protocol for High-Temperature NPIs Treatment. Asobtained NPls were dissolved in $10 \mathrm{~mL}$ of octadecene and transferred into a three-necked flask along with $30 \mu \mathrm{L}(0.1$ mmol) of oleic acid and $80 \mathrm{mg}(0.3 \mathrm{mmol})$ of cadmium acetate. After that, the flask was degassed at room temperature for 15 min, purged with argon, heated to $150{ }^{\circ} \mathrm{C}$, and kept at that temperature for $10 \mathrm{~min}$. After that, the heating was stopped, and flask was allowed to cool. Then, NPls were precipitated out with isopropanol with subsequent centrifugation.

Ligand Exchange. The concentration of the NPls solutions was set at $4 \times 10^{-8} \mathrm{~mol} / \mathrm{L}$ (calculated according to ref 17 ). To induce self-assembly, corresponding amounts of the $3 \times 10^{-3}$ $\mathrm{M}$ solutions of ligands were added to the NPls solution under vigorous stirring. Stirring was continued for $1 \mathrm{~min}$. After that, the solution was transferred into a quartz cuvette and DLS spectra were recorded.

Quantum Yield Determination. Fluorescence quantum yield of nanoplatelets was determined using Rhodamine 6G dye dissolved in ethanol as a reference and calculated in accordance with the expression:

$$
\Phi_{\mathrm{NPls}}=\Phi_{\text {Dye }} \frac{I_{\mathrm{NPls}}}{I_{\text {Dye }}} \frac{\mathrm{OD}_{\text {Dye }}}{\mathrm{OD}_{\text {NPls }}} \frac{n_{\mathrm{NPls}}^{2}}{n_{\text {Dye }}^{2}}
$$

where $\Phi_{\text {Dye }}$ is the Rhodamine $6 \mathrm{G}$ quantum yield $(\Phi=0.94),{ }^{30}$ $I_{\text {NPls }}$ and $I_{\text {Dye }}$ are the integrated intensities of the NPls and dye fluorescence, $\mathrm{OD}_{\mathrm{NPls}}$ and $\mathrm{OD}_{\mathrm{Dye}}$ are the optical densities of the NPls and dye solutions, and $n_{\mathrm{NPls}}$ and $n_{\text {Dye }}$ are the refractive indexes of NPls and dye solutions, respectively. ${ }^{31} \mathrm{PL}$ measurements for QY determination were conducted upon excitation of NPls and dye solutions at $480 \mathrm{~nm}$.

Sample Preparation for IR Spectroscopy. After the addition of ligand solutions, the resulting mixtures were kept for $2 \mathrm{~h}$. After that, NPls were purified by multiple (five times) precipitations and redispersions using chloroform and acetonitrile as solvent and nonsolvent, respectively. After the final precipitation, NPls were dissolved in a small amount of chloroform and drop-cast on the Si plates.

\section{RESULTS AND DISCUSSION}

Current synthesis procedures generally yield colloidal solutions of CdSe NPls that contain both aggregated and individual NPls, as unambiguously demonstrated by TEM and DLS data (Figure 1). Therefore, as a starting point for the current study we 
developed a procedure for the preparation of nonaggregated NPls.

During our preliminary experiments, we noticed that heating of crude 4.5 monolayers thick (4.5 ML) CdSe NPls in chloroform or octadecene in the presence of $\mathrm{Cd}(\mathrm{OAc})_{2}$ or $\mathrm{Zn}(\mathrm{OAc})_{2}$ resulted in the formation of stable colloidal solutions of NPls with increased PL quantum yield (QY). Figure 1 shows TEM images (Figures 1a,b) and DLS distribution curves (Figure 1c) of $4.5 \mathrm{ML} \mathrm{NPls}$ solution prior to and after such treatment with $\mathrm{Cd}(\mathrm{OAc})_{2}$. Prior to treatment, a considerable fraction of NPls is assembled into small stacks with the mean size of several tens of nanometers (Figure 1a), whereas on the TEM image of the treated sample, one can observe only nonaggregated individual NPls that do not form stacks when dried on TEM grid (Figure 1b). On the basis of TEM data, the lateral size of individual NPls was estimated to be $24.3 \pm 0.7$ $\mathrm{nm}$ in length and $8.4 \pm 0.3 \mathrm{~nm}$ in width. (For size distribution, see Figure S1.) According to DLS, prior to treatment NPls are present in the solution in the form of aggregates with a mean size of $28.1 \pm 2.7 \mathrm{~nm}$. At the same time, after the heating of CdSe NPls with cadmium acetate their mean size decreases approximately by a factor of 2 to $11.3 \pm 0.7 \mathrm{~nm}$ (Figure 1c).

With respect to the position of peaks and their relative intensity, absorption spectra (Figure 2) of these two samples

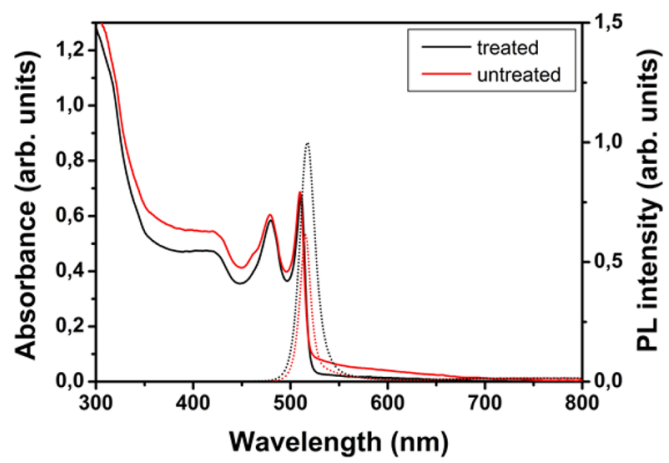

Figure 2. Absorption (solid lines) and PL (dotted lines) spectra of nontreated (red) and cadmium acetate treated (black) 4.5 ML CdSe $\mathrm{NPls}$ in chloroform.

are almost identical except for the presence of the featureless long wavelength tail above $520 \mathrm{~nm}$ attributed to the light scattering by NPls aggregates. Positions of PL peaks also do not differ in these two samples, but PL intensity of nontreated NPls is considerably smaller. Such decreased PL was observed earlier for stacks of NPls formed by the addition of ethanol to their dispersion in hexane ${ }^{28}$ and explained by the efficient FRET in stacks with consecutive exciton trapping by defected NPls.

To study this effect in detail and derive a possible explanation of the observed transformations, we carried out a series of various treatment procedures. Colloidal solution of $4.5 \mathrm{ML}$ CdSe NPls obtained after the synthesis was diluted to the concentration of $4 \times 10^{-8} \mathrm{~mol} / \mathrm{L}$ and divided into eight equal parts. Each part was treated according to Table 1. First, colloidal solutions with different added components were stirred for $0.5 \mathrm{~h}$ and after that were heated at $45^{\circ} \mathrm{C}$ for $2 \mathrm{~h}$ in air. Control sample S7 underwent the same procedure as samples S1-S6 but without any additives to the solution. To distinguish sample transformation due to time and heating, we also introduced a zero-control sample that was kept in dark at
Table 1. Treatment Pattern of Crude 4.5 ML CdSe NPls in Chloroform

\begin{tabular}{ll} 
sample & \multicolumn{1}{c}{ treatment condition } \\
S1 & $0.05 \mathrm{M} \mathrm{Cd}(\text { oleate })_{2}+0.05 \mathrm{M} \mathrm{Cd}(\mathrm{OAc})_{2}$ \\
S2 & $0.05 \mathrm{M} \mathrm{Cd}(\text { oleate })_{2}$ \\
S3 & $0.05 \mathrm{M} \mathrm{Cd}(\mathrm{OAc})_{2}$ \\
S4 & $0.1 \mathrm{M} \mathrm{OA}+0.1 \mathrm{M} \mathrm{NaOAc}$ \\
S5 & $0.1 \mathrm{M} \mathrm{OA}$ \\
S6 & $0.1 \mathrm{M} \mathrm{NaOAc}$ \\
S7 & control sample
\end{tabular}

room temperature. After the treatment, each sample was characterized by optical spectroscopy and DLS measurements.

Neither emission intensity nor the mean diameter of NPls in the control (S7) and zero-control samples changed significantly over time. This allows us to assume that sample changes are generally governed by the composition of the solution. Absorption spectra of samples after heating did not show any differences either prior to or after the heating and were almost identical in all samples. At the same time, PL intensity and average DLS size through the series vary dramatically (Figure 3a).
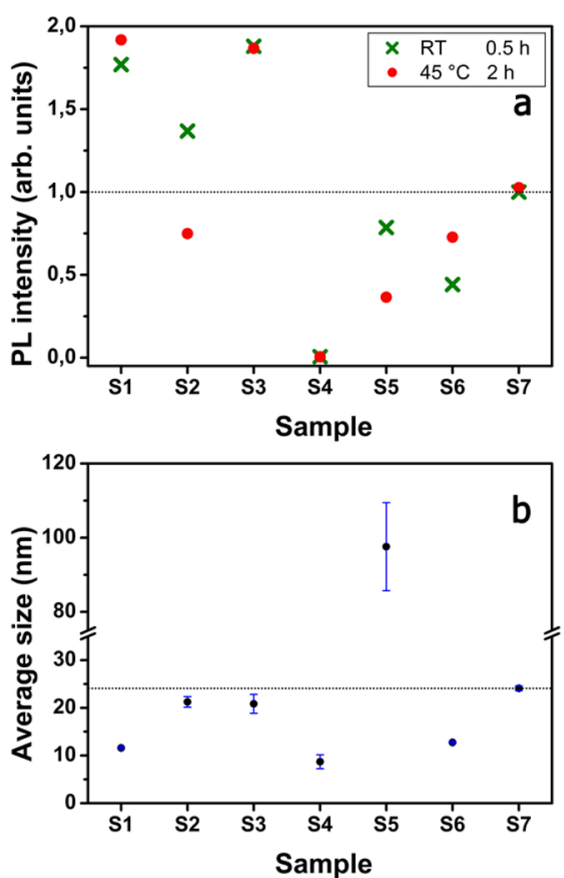

Figure 3. (a) Normalized PL intensity (relatively to S7) of nontreated (S7) and treated (S1-S6) samples of 4.5 ML CdSe NPls in chloroform (green crosses, after $0.5 \mathrm{~h}$ at room temperature; red dots, after the heating at $45{ }^{\circ} \mathrm{C}$ for $2 \mathrm{~h}$ ). (b) Average size of NPls in colloidal solution after the heating for $2 \mathrm{~h}$ at $45{ }^{\circ} \mathrm{C}$ according to DLS. For comparison, initial sizes and PL intensity are indicated by the dashed lines. Scale bars represent $90 \%$ confidence intervals calculated on the basis of three measurements.

Observed changes in PL and average DLS-derived size could be attributed to a variety of factors, which include increase in termination of NPls with cadmium and carboxylate ions and NPls disaggregation.

Both top and bottom basal NPls' sides were shown to be represented by (100) Cd-terminated planes. ${ }^{3,32}$ At the same time, lateral edges are composed of a variety of different other 
planes, and some of them are expected to be nonpolar and Serich ones. Recently, we have shown that passivation of these planes by the overgrowth with $\mathrm{CdS}$ "wings" leads to the increase in PL emission intensity that was attributed to the passivation of lateral sides. ${ }^{6}$ Similarly, NPls treatment with Cd salts may result in the passivation of $\mathrm{Se}$ atoms through the binding of $\mathrm{Cd}$ carboxylates in a Z-type manner. This assumption is also in line with the findings of Anderson et al. that have demonstrated that such binding of $\mathrm{Cd}$ carboxylates has beneficial effect on PL intensity of CdSe QDs. ${ }^{33}$ Because additional $\mathrm{Cd}$ termination occurs only on the lateral edges of $\mathrm{NPls}$, the quantum confinement regime remains unchanged; thus, no changes in the position of both PL and absorption bands are observed.

Considering the facts that both basal planes are terminated with cadmium atoms and that the synthesis procedure involves injection of the relatively large amounts of oleic acid on the final stage, the ligand shell is expected to be composed of acetate and oleate ions with the prevalence of the latter ones. Because basal planes are (100) ZB planes expanded by $2-8 \%$ relative to the bulk value, ${ }^{3,32}$ minimal distance between neighboring cadmium atoms is $0.620-0.657 \mathrm{~nm}$ (in [100] direction) and $0.439-0.464 \mathrm{~nm}$ (in [111] direction). The first is close to the reported distance between two bulky molecules of oleic acid in a self-assembled monolayer $(0.64 \mathrm{~nm}){ }^{34}$ whereas the latter one is nearly 1.5 times smaller. Thus, some of the cadmium atoms may remain unpassivated. Although some additional passivation could be achieved through the binding of oleates in a bridging mode, small acetate ions are also expected to be crucial to provide Cd-passivation and increase ligand coverage of NPls' surface by the formation of the mixed ligand shell. $^{26,35}$ Earlier changes in carboxylate coverage was reported to determine PL emission intensity of CdSe QDs. ${ }^{33}$

Finally, according to DLS measurements (Figure 3b), NPls treatment with acetate ions (samples S1, S3, S4, and S6) regardless of the counterion (sodium or cadmium) leads to their disaggregation. (It should be noted that treatment with $\mathrm{Cd}(\mathrm{OAc})_{2}$ alone results in similar size decrease but takes more time (up to $2 \mathrm{~h}$ ).) Such disaggregation excludes the possibility of the aforementioned FRET in stacked NPls. Combined with the increased $\mathrm{Cd}$ termination, it results in the increased PL intensity observed for NPls treated with $\mathrm{Cd}(\mathrm{OAc})_{2}$ (samples $\mathrm{S} 1$ and S3). In the case when $\mathrm{Cd}$ salts were not present in the solution, PL decrease was observed. Addition of sodium acetate results in rapid and considerable PL quenching, and the nature of such effect is not yet clear.

NPls disaggregation is likely to be explained by the replacement of some of the oleate ions with acetate ones and the dilution of ligand "brush" on the NPls' surface. Such dilution reduces van der Waals interaction ligand-ligand interaction that stabilizes NPls stacks. ${ }^{36}$ In contrast, excess oleic acid (sample S5) displaces acetate ligands and leads to the formation of a denser ligand shell on the NPls surface and to the increase in the number of oleate-oleate contacts, thus favoring stacks formation.

Although S1-treated $\left(\mathrm{Cd}(\mathrm{OA})_{2}+\mathrm{Cd}(\mathrm{OAc})_{2}\right)$ and S3-treated $\left(\mathrm{Cd}(\mathrm{OAc})_{2}\right)$ samples after a prolonged, $10 \mathrm{~h}$ treatment exhibited similar results, both PL intensity and colloidal stability (judging by DLS) of the sample treated with only $\mathrm{Cd}(\mathrm{OAc})_{2}$ were considerably more stable in the time span for at least several months. To make this stabilization method faster and more convenient, we also developed a hightemperature treatment procedure. It involves heating of NPls solution in $\mathrm{ODE}$ with $\mathrm{Cd}(\mathrm{OAc})_{2}$ at $150{ }^{\circ} \mathrm{C}$ for several minutes. (For more details, see the Experimental Section.) Colloidal solution of these individual nonstacked NPls was used for subsequent aggregation experiments.

In the next part of our work, we investigated the assembly of individual NPls under the action of different ligands. Tested ligands include such common molecules for passivation of CdSe NPs as hexadecylphosphonic acid (HDPA), stearic acid (SA), oleic acid (OA), and hexadecanethiol (HDT). All these ligands were reported to bind to the CdSe surface via the $\mathrm{X}$ type bonding between ligand anion and surface $\mathrm{Cd}^{2+}$ ion. ${ }^{37-39}$ Aggregation of nanoparticles can be monitored by a variety of different techniques. In this work, we used dynamic light scattering (DLS) because it allows fast and convenient real-time in situ monitoring of the process. ${ }^{40}$

Figure 4 shows the evolution of the average size of NPls aggregates in time. In the case of HDPA, aggregation starts rapidly and reaches saturation in ca. $10 \mathrm{~min}$ (Figure 4a,b), whereas in the presence of SA, aggregation starts after the
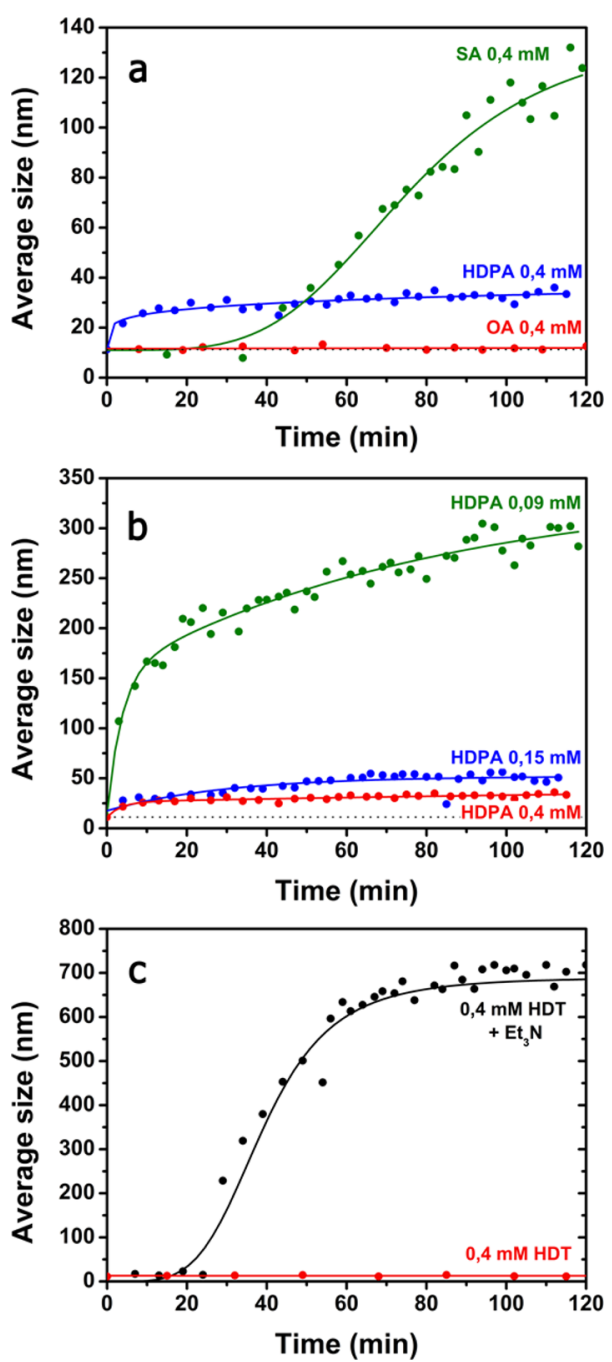

Figure 4. Evolution of the DLS-derived average size of 4.5 ML CdSe $\mathrm{NPls}$ in time in their colloidal solutions in chloroform after the addition of (a) stearic (SA), oleic (OA), and hexadecylphosphonic acids (HDPA) in equimolar concentrations, (b) different concentrations of HDPA, and (c) hexadecanethiol (HDT) in the presence (black) and absence (red) of triethylamine. Dashed lines indicate average size of starting NPls. 
induction period of about $30 \mathrm{~min}$ (Figure 4a). At the same time, addition of the same amount of pure HDT did not induce any aggregation (Figure 4c). However, after $12 \mathrm{~h}$ in the thioltreated sample, small aggregates with the mean size of ca. 30 $\mathrm{nm}$ were observed (Figure S2). In the work of Owen et al., ${ }^{38}$ it was demonstrated that the exchange of anionic species for thiols could be facilitated by the addition of a base such as triethylamine through the formation of a thiolate-ion and a quaternary ammonium salt with acidic anion. Thiolate is a stronger nucleophilic agent than carboxylates and according to calculations binds to $\mathrm{CdSe}$ surface stronger than protonated thiol. $^{22,38,41}$ When HDT was added along with triethylamine into the colloidal solution of acetate-treated CdSe NPls, we observed fast aggregation of NPls after a certain induction period.

We note that aggregation rate (fast aggregation-slow aggregation-no aggregation) and the mean aggregate size in the order HDPA < SA < HDT correlate with the acidity of ligands used for aggregation: $\mathrm{pK}_{\mathrm{a}}(\mathrm{HDPA})=2.6,{ }^{22,42} \mathrm{p} K_{\mathrm{a}}(\mathrm{SA})=$ $4.8-5.6,{ }^{43,44}$ and $\mathrm{pK}$ (alkanethiols $) \approx 10-11 .{ }^{45}$ Earlier it was shown that ligand shell of CdSe quantum dots grown in the presence of oleic acid consists of oleate ions rather than the free acid. $^{37}$ It was also found that oleate ions do not exhibit exchange between bound and free state unless the excess of acidic ligands is added to the solution. ${ }^{37,38}$ Such ligand substitution occurs in a two-step fashion that involves proton exchange between physisorbed acidic species and bound oleate ion. Analogously in the current case of NPls, a similar ligand exchange process can take place driven by a proton exchange reaction. The stronger the acid is, the faster ligand exchange occurs and the higher the supply of monomers for aggregation takes place. It results in rapid aggregation and small size of aggregates. The same trend is observed when the concentration of acid is varied (Figure $4 \mathrm{~b}$ ). A higher concentration of acid leads to fast exchange, which consequently results in smaller size of aggregates.

To verify the accuracy of DLS measurements and study morphology of the aggregates, we acquired TEM images of NPls treated in 0.09 and $0.15 \mathrm{M}$ HDPA solutions for $60 \mathrm{~min}$, i.e., after the size of aggregates reached saturation (Figure $4 b$ ). According to DLS data, these aggregates have an average size of $262.2 \pm 24.7$ and $56.5 \pm 4.6 \mathrm{~nm}$, respectively (Figure 5a,b). It can be seen from Figure $5 \mathrm{c}$ that although the DLS-derived size does not exactly match the TEM-derived size one can still notice pretty good agreement between two techniques, so it may be concluded that DLS can be used to adequately monitor the evolution of NPls stacks size in situ.

For many potential applications, it is highly desired to fabricate stacks of NPls of controlled size. The aggregate size could be varied by the concentration of the added ligands that induce self-assembly. This fact is demonstrated on the Figure 6 on the example of HDPA.

The concentration-dependent curve in Figure 6 shows unusual character with the aggregate size peaking at small HDPA concentrations. Although a small amount of introduced HDPA (below $5 \times 10^{-5} \mathrm{M}$ ) is simply not able to initiate sufficient aggregation, a fast decrease of the mean size of aggregates in the range of $7 \times 10^{-5}-2 \times 10^{-4} \mathrm{M}$ of HDPA can be explained by the mechanism of seed-mediated growth mentioned above. The larger the concentration of the aggregation initiator, e.g., HDPA, present in the colloidal solution, the larger the amount of small NPls aggregates formed
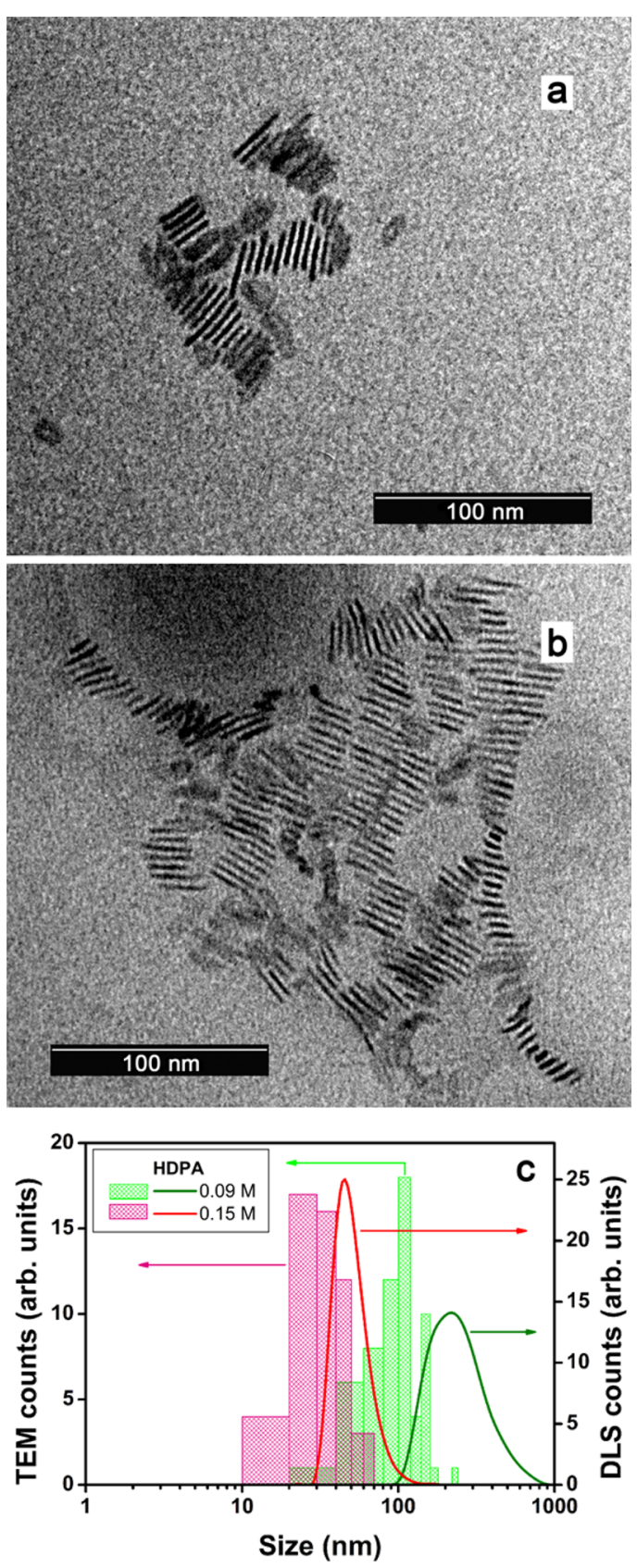

Figure 5. (a and b) TEM images and (c) corresponding DLS curves and size histograms for 4.5 ML CdSe NPls stacks assembled in $0.15 \mathrm{M}$ HDPA (a, red data in panel c) and $0.09 \mathrm{M}$ (b, green data in panel c) solutions in chloroform.

and the smaller the amount of individual NPls remaining in the solution as building blocks.

Surprisingly, TEM images of NPls aggregates formed by the treatment with stearic acid for $1 \mathrm{~h}$ do not show the presence of any stacks (Figure 7a). Instead, NPls lie with their face parallel to the surface of the TEM grid, similar to the case of acetatetreated individual NPls (Figure 1b). However, after the careful comparison of Figures $7 \mathrm{a}$ and $1 \mathrm{~b}$, it can be noticed that after the treatment with stearic acid NPls tend to pack in a side-to-side (tracklike) manner whereas acetate-treated NPls prior to stearic acid exhibit random orientation. One can also notice that NPls are organized into domainlike structure with the domain size of $50-100 \mathrm{~nm}$ that agrees with the DLS data (Figure 7a,b). 


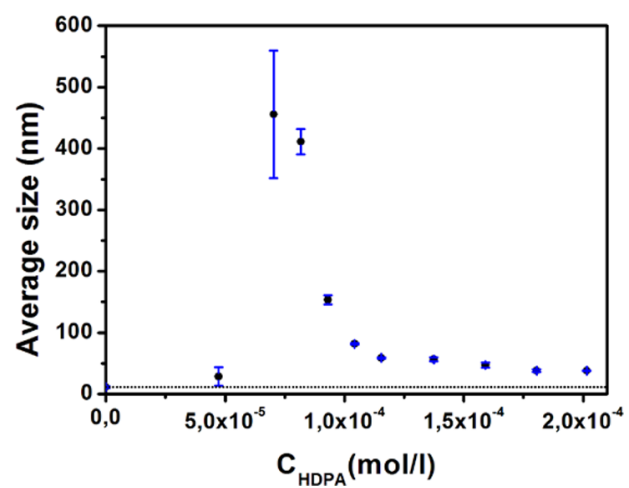

Figure 6. DLS-derived average size of the aggregates of 4.5 ML CdSe NPls in chloroform formed $1 \mathrm{~h}$ after the addition of HDPA at different concentrations. Dashed line at the bottom indicates the average size of $10 \mathrm{~nm}$ of the starting NPls.
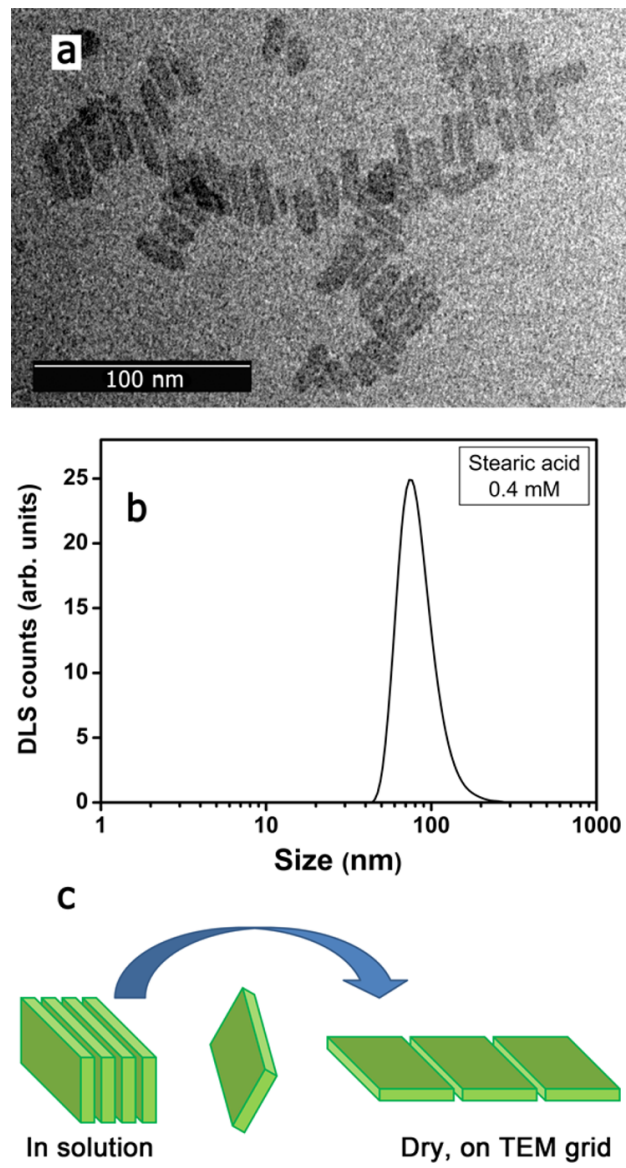

Figure 7. (a) TEM image and (b) DLS curve of CdSe NPls aggregates formed under the action of stearic acid for $1 \mathrm{~h}$; (c) scheme demonstrating dominolike mechanism of disaggregation of SAstabilized NPls on TEM grid.

It is highly unlikely that such lamellar aggregates with the side-to-side arrangement can be formed in the solution. Recently, Jana et al. demonstrated the formation of stacks of CdSe NPls in colloidal solutions after the ligand exchange with different fatty acids. ${ }^{36}$ Because SA induces the efficient aggregation of CdSe NPls but the same amount of oleic acid under similar conditions does not produce aggregation (Figure $4 \mathrm{a})$, the presence of dense brush of rigid saturated alkyl SA chains on the surface of NPls can be crucial for the formation of the weakly bound NPls stacks. If stacks of SA-stabilized CdSe $\mathrm{NPls}$ are only weakly bound, then they could easily disaggregate during the drying on the substrate or TEM grid in a dominolike mechanism depicted in Figure $7 \mathrm{c}$.

Efficiency of ligand exchange with carboxylic and phosphonic acids and hexadecanethiol was also examined by IR spectroscopy. Corresponding IR spectra of purified ligand-exchanged NPls are shown on Figure 8. A group of bands around 2800-

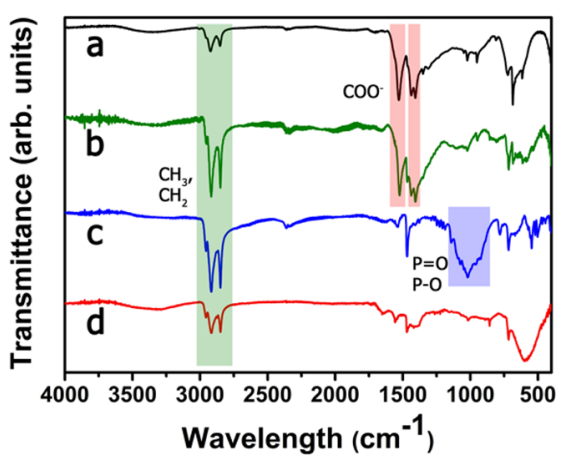

Figure 8. IR spectra of (a) starting OA-capped 4.5 ML CdSe NPls, and NPls after the ligand exchange with (b) SA, (c) HDPA, and (d) $\mathrm{HDT} / \mathrm{Et}_{3} \mathrm{~N}$ solutions.

$3000 \mathrm{~cm}^{-1}$ present on all spectra is a characteristic feature for molecules containing long alkyl chains. These bands are assigned to symmetric $\left(\nu_{\mathrm{s}}\left(\mathrm{CH}_{3}\right)=2870 \mathrm{~cm}^{-1} ; \nu_{\mathrm{s}}\left(\mathrm{CH}_{2}\right)=\right.$ $\left.2848-2852 \mathrm{~cm}^{-1}\right)$ and antisymmetric $\left(\nu_{\text {as }}\left(\mathrm{CH}_{3}\right)=2950-2955\right.$ $\left.\mathrm{cm}^{-1} ; \nu_{\text {as }}\left(\mathrm{CH}_{2}\right)=2916-2921 \mathrm{~cm}^{-1}\right)$ vibrations of methyl and methylene units. ${ }^{46,47}$

On the spectra of OA- and SA-capped NPls (Figure 8 a,b), another group of bands is present at $1400-1550 \mathrm{~cm}^{-1}$. These features are characteristic for carboxylate group and consist of bands peaking at $1523-1529 \mathrm{~cm}^{-1}\left(\nu_{\text {as }}\left(\mathrm{COO}^{-}\right)\right), 1436-1437$ $\left.\mathrm{cm}^{-1}\left(\nu_{\mathrm{s}}\left(\mathrm{COO}^{-}\right)\right)\right)^{48}$ and $1405-1406 \mathrm{~cm}^{-1}$ (scissoring vibration of $\alpha$-methylene group of carboxylic acids $)^{46}$ for SAand OA-capped NPls, respectively. The rather low value of separation of asymmetric and symmetric vibrations $(\Delta v \approx 90$ $\mathrm{cm}^{-1}$ ) suggests that carboxylates form bidentate chelate bonds with the surface. 48,49

Upon addition of HDPA and $\mathrm{HDT} / \mathrm{Et}_{3} \mathrm{~N}$ (Figure $8 \mathrm{c}, \mathrm{d}$ ), these bands disappear almost completely (band peaking at 1469 $\mathrm{cm}^{-1}$ attributed to scissoring vibration of methylene unit in alkyl chain), ${ }^{46}$ thus indicating that addition of HDPA or HDT/ $\mathrm{Et}_{3} \mathrm{~N}$ results in the displacement of oleate ions. This conclusion is also supported by the appearance of new broad mixed bands at $900-1150$ and $500-750 \mathrm{~cm}^{-1}$ that can be attributed to the variety of valence and deformation vibrations of $\mathrm{P}-\mathrm{O}, \mathrm{P}=\mathrm{O}$, and $\mathrm{C}-\mathrm{S}$ bonds in the case of HDPA and $\mathrm{HDT} / \mathrm{Et}_{3} \mathrm{~N}$, respectively. ${ }^{47}$

Closer examination of IR spectra also allows to gain some information on the spatial arrangement of the ligand layer. IR spectroscopy is widely used to study ordering of alkyl chains in self-assembled monolayers, ${ }^{50-52}$ ligand layers on the NPs' surface, ${ }^{53,54}$ or their orientational disordering upon melting of lipid membranes. ${ }^{46}$ Figure 9a shows that upon exchange of oleic acid for SA, HDPA, or HDT with saturated alkyl chains a new group of bands appears at $1150-1400 \mathrm{~cm}^{-1}$. This group is attributed to methylene wagging and twisting vibrations and appears when the entire alkyl chain is in the zigzag all-trans conformation without gauche defects. ${ }^{51}$ Number and spacing of these bands depend on the length and conformation of the 

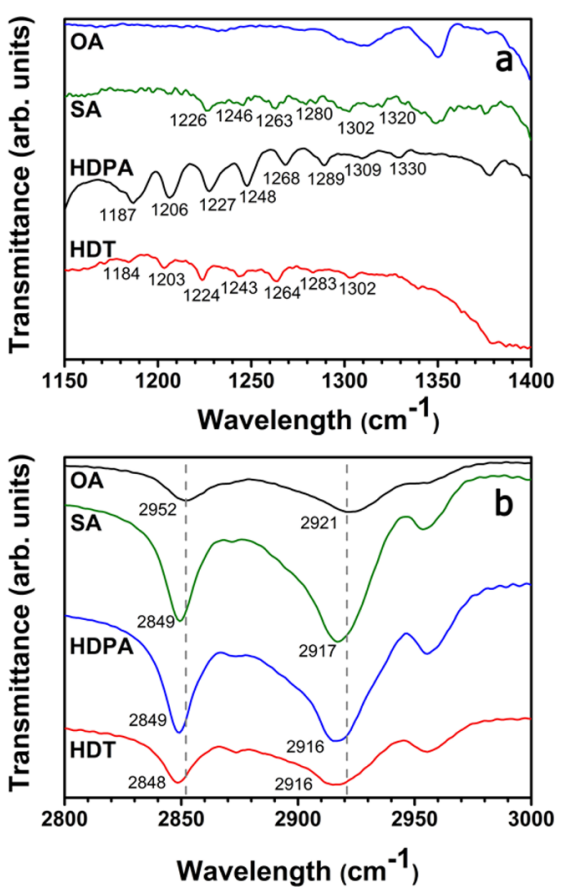

Figure 9. IR spectra of $4.5 \mathrm{ML}$ CdSe NPls capped with OA, SA, HDPA, and HDT in the regions of (a) $1150-1400 \mathrm{~cm}^{-1}$ and (b) $2800-3000 \mathrm{~cm}^{-1}$ demonstrating vibration of alkyl chain units.

alkyl chain. Spacing between bands could be calculated according to $\Delta v=326 /(m+1)$, where $m$ is the number of methylene units. For HDPA and HDT, $m=15$, whereas for SA, $m=17$. Thus, one should expect spacing of 20.4 and $18.1 \mathrm{~cm}^{-1}$, respectively. Using this equation and the experimental data, we obtained $\Delta v$ values of $20.43 \pm 0.58,19.67 \pm 0.85$, and $18.80 \pm$ $2.07 \mathrm{~cm}^{-1}$ for HDPA, HDT, and SA, respectively. These findings agree well with the predicted calculated values. The number of these bands can be calculated by the formula $N=(m$ $+1) / 2$. Thus, we should observe 8 (HDPA and HDT) and 9 (SA) peaks for methylene wagging vibrations. Because of the intrinsic low intensities of these vibrations, oftentimes not all bands are observed. ${ }^{46,51}$ However, in the case of HDPA the densest chain packing can be achieved, which is due to the fact that HDPA is the most acidic compound among studied ligands and exchange is fast and almost complete. In the case of SA and HDT, less denser packing is achieved possibly because of some remaining oleic acid that introduces disorder into the ligand layer. Presence of this pattern in the SA-treated sample also allows us to assume that at least some $\mathrm{OA}$ is displaced from NPls' surface by SA (Figure 9a).

Chain-packing density and chain conformation also influences position of the bands corresponding to the valence vibrations of alkyl chains at $2800-3000 \mathrm{~cm}^{-1}$ (Figure 9b). ${ }^{46,50,51}$ Ordering of alkyl chains results in the shift of signals of both symmetric and asymmetric vibrations of methylene units from 2852 to $2848-2849 \mathrm{~cm}^{-1}\left(\nu_{\mathrm{s}}\left(\mathrm{CH}_{2}\right)\right)$ and from 2921 to $2917-2916 \mathrm{~cm}^{-1}\left(\nu_{\text {as }}\left(\mathrm{CH}_{2}\right)\right)$ for NPls capped with $\mathrm{OA}$ and ligands with saturated chains, respectively. It further supports the idea of the formation of close-packed layer of all-trans-saturated alkyl chains on the NPls' surface after the ligand exchange.

The self-assembly process can be understood as the interplay of forces of different nature. Among the most frequently considered ones are van der Waals, electrostatic, and steric forces. Previously reported and current assembly procedures of NPls stacking seem to be driven by the same van der Waals attraction force between two flat plates with the consequent stabilization of stacks by the interchain interaction. At the same time, assembly processes supposedly proceed through different pathways. Addition of alcohol as a "bad" solvent results in the shrinkage of the ligand layer that decreases the value of the steric repulsion potential and allows a closer approach of the NPls to each other, thus increasing the van der Waals attractive potential. $^{26,55}$ Quantum calculations also show that in the presence of polar solvents strongly repulsive interaction between ligand chains becomes attractive. ${ }^{56}$

In our case, no significant changes in solvent polarity occur so that hydrocarbon chains remain stretched from the surface forming ligand "brush" on the NPls' surface. When NPls collide with each other because of Brownian motion, such polymer brushes can interpenetrate each other, and ligand chains attractively interact through dispersive forces. ${ }^{55,57}$ Such dispersive forces, usually referred to as hydrophobic interaction, are widely employed for water solubilization of hydrophobic NPs in polar solvents using amphiphilic polymers. Efficient polymer overcoating in this case is achieved through the multiple interactions of side alkyl branches of the polymer chain with the alkyl chains of ligands attached to the NPs' surface. ${ }^{58-62}$ Analogously, NPls stacks are stabilized by multiple hydrophobic interactions between ligand chains of neighboring NPls. In comparison with QDs, NPls possess very high surface area; thus, the area of interaction becomes very large, which leads to a more efficient aggregation in the case of NPls.

Another factor that affects NPls' aggregation is the local surface curvature. Surface curvature (particle radius) has a great impact on the ligand-particle interface and interparticle interaction that manifests in differences in the ligand-packing density, ${ }^{21,26,63,64} \mathrm{pK}_{\mathrm{a}}$ of adsorbed ligands, ${ }^{65}$ self-assembly, ${ }^{55,66}$ and so on. Unlike QDs, NPls have lower surface curvature that allows hydrocarbon chains overlap more effectively, similar to the case of large gold spheres and flat prisms. ${ }^{66}$

High surface area with low curvature and hence a large number of dispersive contacts between chains may result in the increased interchain interaction coupled with the van der Waals attraction between two flat plates, becoming a driving force for the assembly of NPls into stacks. At the same time, upon close approach steric repulsion becomes more and more significant. Thus, self-assembly is governed by the balance of the van der Waals attraction of two nanoplatelets and dispersive interaction of hydrocarbon chains on one hand and repulsive steric potential on the other. Our experiments suggest that this balance could be manipulated by the NPls surface ligands nature.

As it was described above, upon the treatment of NPls with $\mathrm{Cd}(\mathrm{OAc})_{2}$, acetate ions displace surface-bound oleate ions and dilute the polymer brush on the NPls' surface, thus decreasing the number of dispersive contacts that stabilize a NPls stack (Figure 10a,b). At the same time, repulsive steric potential seems to be less susceptible to the changes in the ligand shell density, and after the acetate treatment, it overpowers both attractive forces.

Exchange of the oleic acid containing soft curved alkenyl chain for ligands with straight saturated chains results in denser polymer brush on the NPl's surface, which gives more efficient dispersive contact in the interpenetrated brushes (Figure 10c). For the calculation of the energy of dispersive attraction between alkyl chains, Salem ${ }^{55}$ suggested the equation: 


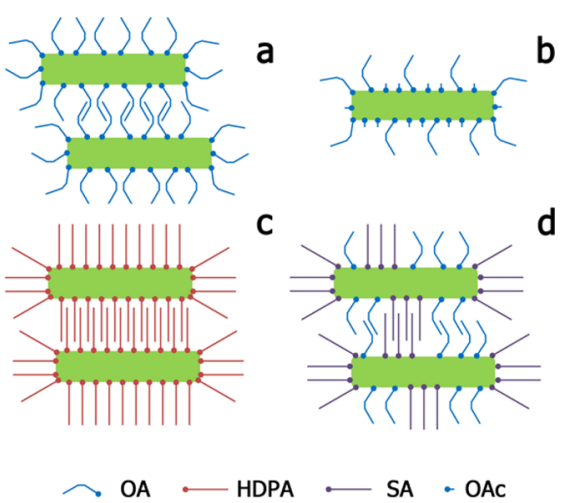

Figure 10. Schematic depiction of the ligand layers on NPls (a) before and (b) after the treatment with Cd acetate and after ligand exchange with (c) HDPA and (d) SA.

$$
E=A \frac{3 \pi}{8 \lambda^{2}} \frac{L}{D^{5}}
$$

where $A$ is the Hamaker constant, $\lambda$ is the length of the basic unit (methylene unit, $0.127 \mathrm{~nm}$ ), $L$ is the length of molecular overlap, and $D$ is the close-packing separation distance.

As one can see, the interaction energy strongly depends on the separation distance of alkyl chains $(D)$. For oleic acid, $D$ is determined by the size of the alkenyl group in cis conformation, whereas in the case of ligands with saturated chains, this distance mainly depends on the headgroup size. It can be demonstrated by comparing molecule footprints and limiting areas of molecules in self-assembled monolayers. Oleic acid has a footprint of 41-50 $\AA^{2}$, whereas HDPA, SA, and HDT are characterized by smaller footprints of $25-28,20$, and $21 \AA^{2}$ (saturated alkyl chain footprint $=17-18 \AA^{2}$ ), respectively. ${ }^{34,51,67-69}$ Thus, two ligand brushes formed by saturated chains on the flat surfaces can create stronger attractive potential because of a closer approach of the interacting chains. Moreover, because of the curved hydrocarbon tail, oleic acid creates weak chain-to-chain contacts with a lower value of the effective interaction length $L$ that further decreases the overall attraction energy (Figure 10a,c).

Formation of the loosely bound NPls stacks upon their treatment with stearic acid can be attributed to the formation of the mixed SA-OA ligand shell (Figure $10 \mathrm{~d}$ ) because of the fact that the $\mathrm{p} K_{\mathrm{a}}$ values for SA and OA are very close $(\sim 5)$ and that ligand exchange thus occurs slowly and selectively. Such a mixed shell is expected to form less-effective dispersion contacts than those of a ligand shell consisting of one type of ligands. Because carboxylate exchange is a two-step process that involves a psysisorption stage, the steric availability of binding sites may play important role. The highly curved surface of NPls edges may afford more efficient absorption because of the less sterically hindered surface. ${ }^{21,26}$ Additionally, Boles and Talapin $^{55}$ have recently showed that tetrahedral NPs tend to assemble vertex-to-vertex rather than in a face-to-face manner. According to the Flory-Huggins model implemented in that study, flat surfaces (tetrahedron faces) experience steric repulsion considerably larger than that of highly curved surfaces (tetrahedron vertexes), which makes vertex-to-vertex contacts more preferable. Similarly, it can be inferred that such preferable attachment along with other factors explains the formation of the tracklike domain structure observed on the TEM-images of SA-treated NPls.

We also neglect the role of the depletion attraction force because self-assembly by the depletion attraction requires concentrations of additives and nanoparticles (at least 0.5 and 1 $\times 10^{-6} \mathrm{M}$, respectively) higher than those we used in our experiments. ${ }^{40,70}$ To rule out completely the depletion attraction component, we carried out additional experiments
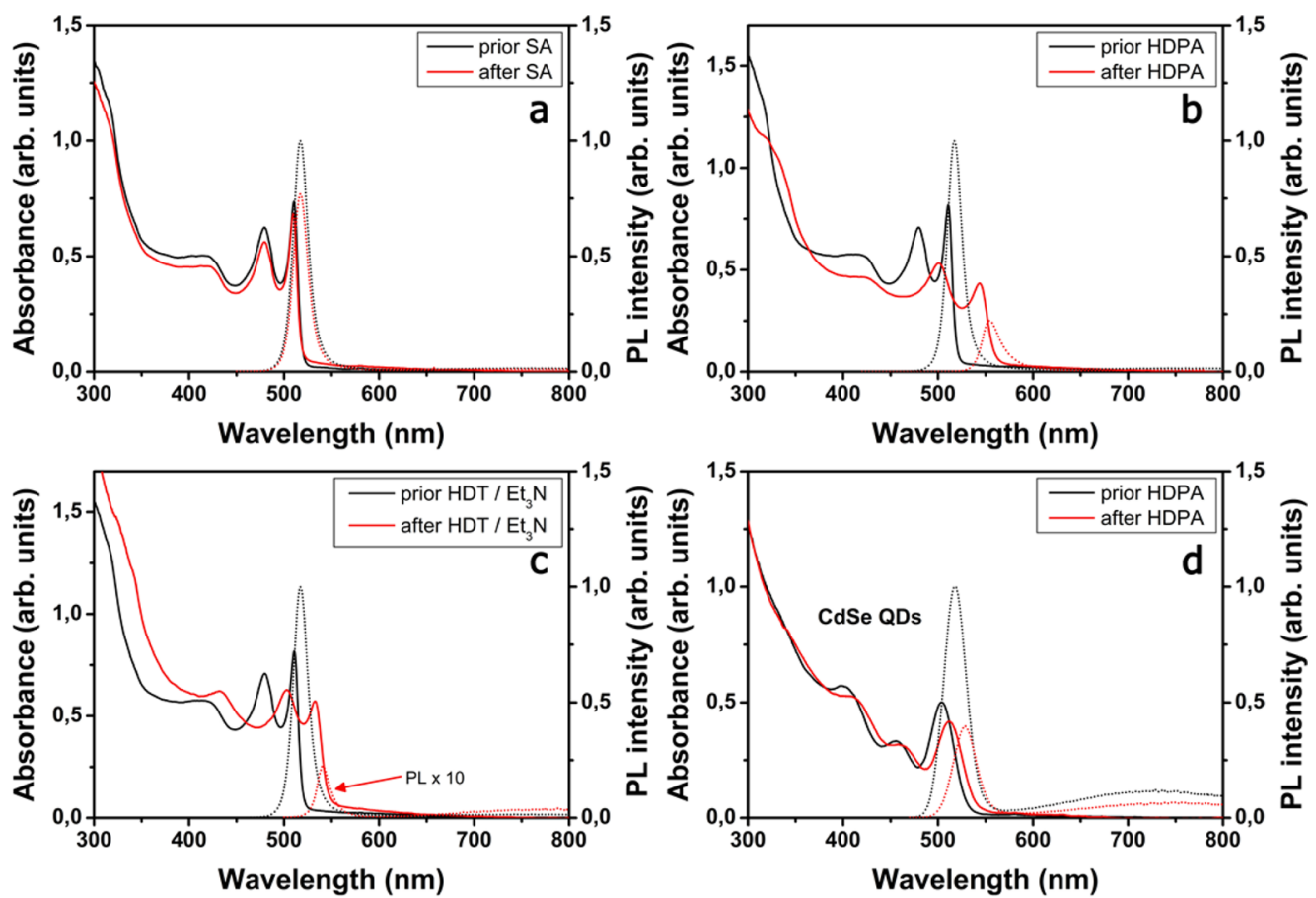

Figure 11. Absorption (solid lines) and PL (dotted lines, $\lambda_{\text {exc }}=430 \mathrm{~nm}$ ) spectra of $(\mathrm{a}-\mathrm{c}) 4.5 \mathrm{ML}$ CdSe NPls and (d) $2.5 \mathrm{~nm}$ CdSe zinc blende QDs prior to (black) and after (red) the ligand exchange with (a) stearic and (b and d) hexadecylphosphonic acids and (c) hexadecanethiol/Et ${ }_{3} \mathrm{~N}$ mixture. 
with high concentrations of oleic acid, hexadecanol, and docosane in the NPls' solution. The last two molecules do not interact with the surface $\mathrm{Cd}$ atoms and do not displace oleate ions from the NPls' surface, thus serving as long-chain inert diluents. The addition of oleic acid to a final concentration of $0.3 \mathrm{M}$ (the same concentration as used in the preparation step) resulted in the formation of NPls stacks of $20-40 \mathrm{~nm}$ in size, which was similar to the value for the nontreated sample (Figure S3). At the same time, the addition of hexadecanol and docosane at even higher concentrations (up to $0.5 \mathrm{M}$ ) did not cause any detectable changes in the mean size and mean count rate (Figure S4). This observation allows us to exclude the depletion attraction at least for the NPls concentration used in our experiments. Different action of the oleic acid may be accounted to the proton exchange and displacement of the acetate ions from the NPls surface.

Finally, we discuss changes in optical properties of $4.5 \mathrm{ML}$ CdSe NPls during the ligand exchange and stacking aggregation. Figure 11 shows optical absorption and PL spectra of colloidal solutions of the acetate-treated 4.5 ML CdSe NPls prior to and after the ligand exchange with SA, HDPA, and HDT.

The ligand exchange of oleic to stearic acid (Figure 11a) did not induce any changes in optical spectra because both acids have the same headgroup with comparable dipole moment $(\sim 1.8 \mathrm{D}) .^{71}$ In the case of alkylphosphonic acid, we observed considerable red-shifting of both absorption and PL bands accompanied by spectral broadening (Figure 11b). Here, the phosphonic acid headgroup has a significantly larger dipole moment $(>2.5 \mathrm{D}) .{ }^{72}$ At the same time, the addition of the same amount of thiol did not induce any shift of absorption peak within $2 \mathrm{~h}$ but rather resulted in nearly complete PL quenching (Figure S5). A redshift of absorption bands was observed only after $12 \mathrm{~h}$. On the contrary, the addition of triethylamine to the NPls solution with $0.4 \mathrm{mM}$ HDT resulted in a fast $20 \mathrm{~nm}$ redshift of absorption band accompanied by strong PL quenching (Figure 11c). Such a redshift is close to the one reported earlier for 4.5 ML CdSe NPls treated with dodecanethiol. $^{4}$ Our observation is consistent with the assumption made above that protonated thiol does not displace oleate ions on CdSe surface and is also supported by comparison of IR spectra of NPls treated with HDT and $\mathrm{HDT} / \mathrm{Et}_{3} \mathrm{~N}$ mixture (Figure S6). A similar effect of thiols on the optical spectra is well-documented for colloidal QDs and can be attributed to the changes of the local dielectric environment as well as the intrinsic dipole moments of the ligands. (Dipole moment of the thiol headgroup $(2.24 \mathrm{D})^{73}$ is larger than that of stearic acid.) It becomes especially important for CdSe NPls because they are in the regime of strong transverse quantum confinement. ${ }^{13}$ The large magnitude of the redshift and spectral broadening in the case of HDPA in comparison with HDT was not reported before. A similar effect was also observed for zinc-blende CdSe QDs $2.5 \mathrm{~nm}$ in diameter dispersed in chloroform (Figure 11d).

Comparing the spectral shifts and the dipole moments, we see that the observed Stokes shift increases with increasing ligand dipole moment. This is a result of the polarization of the exciton due to the ligands' permanent dipole moments, resulting in a reduced electron and hole wave function overlap and hence a reduced transition oscillator strength. ${ }^{13,26,74}$ These distorted electron and hole wave functions have a stronger dipolar character and hence couple more strongly to lattice phonons. This resulting stronger phonon coupling is also evidenced by the fact that with the most polar HDPA ligands we observe the largest Stokes shift with respect to the other ligands both for the $\mathrm{CdSe}$ dots and platelets treated with HDPA and also the largest overall redshift.

Another possible reason for the observations above may be that the ligand footprints in Figure $11 \mathrm{~b}, \mathrm{c}$ do not match the density of terminal $\mathrm{Cd}$ atoms. Hence, the surface density of ligands may be too low, and the effective dielectric constant of the effective ligand medium is lowered below the dielectric constant value of the densest ligand packing according to the footprints discussed above. If the surrounding dielectric constant is lowered, then it results in an increase of the exciton binding energy and a transition redshift. ${ }^{13}$ Additional DFT calculations are already scheduled in order to determine the influence of different surface groups on the energy levels of semiconductor nanostructures in the strong quantum confinement regime because of their donor-acceptor, redox, and dipolar character.

The influence of ligand exchange on the optical properties of NPls was also characterized by time-resolved measurements. Figure 12 shows PL decay curves for stacked CdSe NPls (black curve) and NPls treated with $\mathrm{Cd}(\mathrm{OAc})_{2}$ (green curve) shown in the TEM images on Figure 1a,b, respectively.

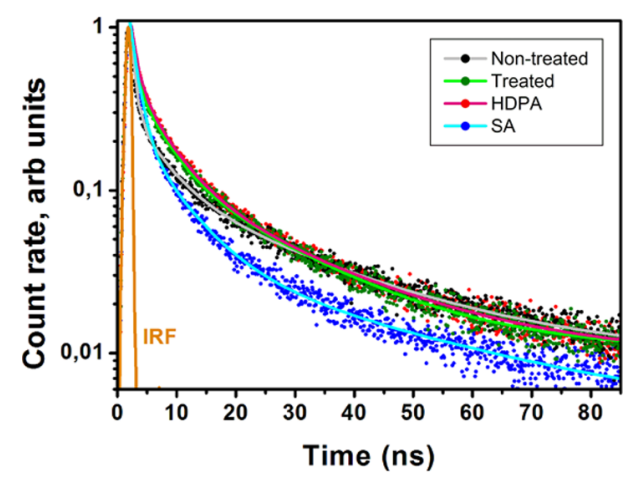

Figure 12. PL decay curves of crude, partially aggregated 4.5 ML CdSe NPls in chloroform (black), S3-treated (Table 1) nonaggregated NPls prior to (green) and after the ligand exchange with hexadecylphosphonic acid (HDPA, red) and stearic acid (SA, blue).

Table 2 presents parameters for PL decay curves in Figure 12 derived from two-exponential fitting of the decay curves.

Disaggregation of NPls ensembles by the additional treatment with $\mathrm{Cd}(\mathrm{OAc})_{2}$ affects their PL decay times in colloidal solution. It can be seen that the acetate-treated CdSe NPls are characterized by a slow early decay component (5-20 ns) and an increased PL quantum yield as compared to those of nontreated NPls. Nontreated, partially stacked NPls show a

Table 2. Decay Times $\tau$, Weighting Coefficients $A$, and PL Quantum Yields QY in Chloroform for Crude Nontreated 4.5 ML CdSe NPls and Acetate-Treated NPls before and after the Ligand-Exchange Procedure with HDPA and SA

\begin{tabular}{lrcccc}
\multicolumn{1}{c}{ sample } & $\mathrm{QY}(\%)^{a}$ & $\tau_{1}(\mathrm{~ns})$ & $A_{1}$ & $\tau_{2}(\mathrm{~ns})$ & $A_{2}$ \\
nontreated & 10.1 & $1.7 \pm 0.03$ & 2.5 & $15.3 \pm 0.2$ & 0.2 \\
acetate-treated & 25.0 & $2.0 \pm 0.04$ & 1.8 & $12.6 \pm 0.1$ & 0.3 \\
HDPA-treated & 6.3 & $1.9 \pm 0.03$ & 3.2 & $12.0 \pm 0.1$ & 0.3 \\
SA-treated & 26.7 & $1.4 \pm 0.01$ & 4.6 & $10.0 \pm 0.1$ & 0.2
\end{tabular}

${ }^{a}$ Average values measured with $95 \%$ precision relatively to Rhodamine $6 \mathrm{G}$. 
faster short decay time and a slower long decay component. All these features can be attributed to the efficient FRET in stacks with exciton migration to the defected NPls where it is trapped and decays nonradiatively. ${ }^{28}$

Despite strong changes in absorption and PL spectra of 4.5 ML CdSe NPls after HDPA-induced aggregation, the character of the corresponding PL decay curve is close to that of acetatetreated individual NPls (Figure 12) with a considerably lower QY yield of the HDPA-treated NPls (Table 2). SA-treated NPls exhibit the fastest PL decay among all studied samples except for HDT because of its strong PL quenching. If one assumes that FRET in NPls stacks plays a major role in the changes of the PL quantum yield and decay times, then the SA-assembled NPls stacks should demonstrate the most efficient FRET because of an almost unaffected PL QY and strong spectral overlap of first excitonic absorption and PL bands (Figure 11a). HDPA-induced stacks possess a lower PL QY (Table 2) and at the same time a lower spectral overlap (Figure 11b), implying a lower FRET effect. Because phosphonic groups bound to surface Cd atoms of CdSe NPls strongly affect the energy levels (according to Figure 11b), the exciton decay dynamics may be also altered toward longer lifetimes. Thereby, weaker FRET and longer life times can result in longer PL decay times for HDPA-induced aggregates (Figure 12).

\section{CONCLUSIONS}

The ligand exchange process on the surface of CdSe nanoplatelets is a versatile method for the formation of selfassembled nanoplatelets' stacks of controlled size. Among different studied ligands, alkylphosphonic acid was shown to be the most suitable agent because it allows control of the average size (length) of the NPls stacks in a broad range by varying its concentration and reaction time. We have also shown that NPls' aggregation kinetics could be simply and accurately studied in situ with the help of dynamic light scattering. The mechanism that governs controlled formation of NPls stacks involves strong van der Waals interaction between alkyl chains brushes on the surface of neighboring NPls. Interaction strength and consequently length and colloidal stability of stacks was shown to be dependent on the type and concentration of different ligands. Colloidal stability of NPls can be achieved by the acetate treatment of oleate-capped NPls, thus indicating the crucial role of the curved oleic alkyl chains and ligand brush dilution for the formation of colloidal solutions of individual nonaggregated CdSe NPls in chloroform. Exchange for straight-chain hexadecylphosohonic acid, which strongly binds to the surface $\mathrm{Cd}$ atoms, results in stronger van der Waals interaction and fast NPls aggregation into longer stacks. We believe that the developed technique can be further extended to the formation of heteroaggregates of $\mathrm{NPls}$ of different thickness (quantum confinement) or even different materials ( $\mathrm{CdSe}, \mathrm{CdS}$, and $\mathrm{CdTe}$ ), thus opening the way for new materials and superstructures with unique optical properties.

\section{ASSOCIATED CONTENT}

\section{S Supporting Information}

The Supporting Information is available free of charge on the ACS Publications website at DOI: 10.1021/acs.jpcc.5b12139.

TEM size distribution histograms of individual NPls, additional absorption and IR spectra, and DLS size distribution curves. (PDF)

\section{AUTHOR INFORMATION}

\section{Corresponding Author}

*E-mail: m_artemyev@yahoo.com.

\section{Present Address}

A. Achtstein: Chemical Engineering Department, Delft University of Technology, Julianalaan 136, 2628 BL Delft, The Netherlands.

\section{Notes}

The authors declare no competing financial interest.

\section{ACKNOWLEDGMENTS}

A. Antanovich, A.P., and M.A. acknowledge CHEMREAGENTS program for financial support. A. Achtstein acknowledges the financial support from DFG Grant AC290/1. We would like to thank L. Gurinovich and A. Stupak for help with PL decay time measurements, A. Karoza for help with IR spectroscopy, and K. Skrotskaya for TEM investigation.

\section{REFERENCES}

(1) Ithurria, S.; Dubertret, B. Quasi 2D Colloidal CdSe Platelets with Thicknesses Controlled at the Atomic Level. J. Am. Chem. Soc. 2008, 130, 16504-16505.

(2) Li, Z.; Qin, H.; Guzun, D.; Benamara, M.; Salamo, G.; Peng, X. Uniform Thickness and Colloidal-Stable CdS Quantum Disks with Tunable Thickness: Synthesis and Properties. Nano Res. 2012, 5, 337351.

(3) Li, Z.; Peng, X. Size/shape-Controlled Synthesis of Colloidal CdSe Quantum Disks: Ligand and Temperature Effects. J. Am. Chem. Soc. 2011, 133, 6578-6586.

(4) Mahler, B.; Nadal, B.; Bouet, C.; Patriarche, G.; Dubertret, B. Core/shell Colloidal Semiconductor Nanoplatelets. J. Am. Chem. Soc. 2012, 134, 18591-18598.

(5) Ithurria, S.; Talapin, D. V. Colloidal Atomic Layer Deposition (cALD) Using Self-Limiting Reactions at Nanocrystal Surface Coupled to Phase Transfer between Polar and Nonpolar Media. J. Am. Chem. Soc. 2012, 134, 18585-18590.

(6) Prudnikau, A.; Chuvilin, A.; Artemyev, M. V. CdSe-CdS Nanoheteroplatelets with Efficient Photoexcitation of Central CdSe Region through Epitaxially Grown CdS Wings. J. Am. Chem. Soc. 2013, 135, 14476-14479.

(7) Tessier, M. D.; Spinicelli, P.; Dupont, D.; Patriarche, G.; Ithurria, S.; Dubertret, B. Efficient Exciton Concentrators Built from Colloidal Core/crown CdSe/CdS Semiconductor Nanoplatelets. Nano Lett. 2014, 14, 207-213.

(8) Antanovich, A. V.; Prudnikau, A. V.; Melnikau, D.; Rakovich, Y. P.; Chuvilin, A.; Woggon, U.; Achtstein, A. W.; Artemyev, M. V. Colloidal Synthesis and Optical Properties of Type-II CdSe-CdTe and Inverted $\mathrm{CdTe}-\mathrm{CdSe}$ Core-wing Heteronanoplatelets. Nanoscale 2015, 7, 8084-8092.

(9) Pedetti, S.; Ithurria, S.; Heuclin, H.; Patriarche, G.; Dubertret, B. Type-II Core/Crown CdSe/CdTe Semiconductor Nanoplatelets. J. Am. Chem. Soc. 2014, 136, 16430-16438.

(10) Kelestemur, Y.; Olutas, M.; Delikanli, S.; Guzelturk, B.; Akgul, M. Z.; Demir, H. V. Type-II Colloidal Quantum Wells: CdSe/CdTe Core/Crown Heteronanoplatelets. J. Phys. Chem. C 2015, 119, 21772185.

(11) Wu, K.; Li, Q.; Jia, Y.; McBride, J. R.; Xie, Z.; Lian, T. Efficient and Ultrafast Formation of Long-Lived Charge-Transfer Exciton State in Atomically Thin Cadmium Selenide/Cadmium Telluride Type-II Heteronanosheets. ACS Nano 2015, 9, 961-968.

(12) Ithurria, S.; Tessier, M. D.; Mahler, B.; Lobo, R. P. S. M.; Dubertret, B.; Efros, A. L. Colloidal Nanoplatelets with TwoDimensional Electronic Structure. Nat. Mater. 2011, 10, 936-941.

(13) Achtstein, A. W.; Schliwa, A.; Prudnikau, A.; Hardzei, M.; Artemyev, M. V.; Thomsen, C.; Woggon, U. Electronic Structure and 
Exciton-Phonon Interaction in Two-Dimensional Colloidal CdSe Nanosheets. Nano Lett. 2012, 12, 3151-3157.

(14) She, C.; Fedin, I.; Dolzhnikov, D. S.; Demortière, A.; Schaller, R. D.; Pelton, M.; Talapin, D. V. Low-Threshold Stimulated Emission Using Colloidal Quantum Wells. Nano Lett. 2014, 14, 2772-2777.

(15) Grim, J. Q.; Christodoulou, S.; Di Stasio, F.; Krahne, R.; Cingolani, R.; Manna, L.; Moreels, I. Continuous-Wave Biexciton Lasing at Room Temperature Using Solution-Processed Quantum Wells. Nat. Nanotechnol. 2014, 9, 891-895.

(16) She, C.; Fedin, I.; Dolzhnikov, D. S.; Dahlberg, P. D.; Engel, G. S.; Schaller, R. D.; Talapin, D. V. Red, Yellow, Green, and Blue Amplified Spontaneous Emission and Lasing Using Colloidal CdSe Nanoplatelets. ACS Nano 2015, 9, 9475-9485.

(17) Achtstein, A. W.; Antanovich, A.; Prudnikau, A.; Scott, R.; Woggon, U.; Artemyev, M. Linear Absorption in CdSe Nanoplates: Thickness and Lateral Size Dependency of the Intrinsic Absorption. J. Phys. Chem. C 2015, 119, 20156-20161.

(18) Olutas, M.; Guzelturk, B.; Kelestemur, Y.; Yeltik, A.; Delikanli, S.; Demir, H. V. Lateral Size-Dependent Spontaneous and Stimulated Lateral Size-Dependent Spontaneous and Stimulated Emission Properties in Colloidal CdSe Nanoplatelets. ACS Nano 2015, 9, 5041-5050.

(19) Scott, R.; Achtstein, A. W.; Prudnikau, A.; Antanovich, A.; Christodoulou, S.; Moreels, I.; Artemyev, M.; Woggon, U. Two Photon Absorption in II-VI Semiconductors: The Influence of Dimensionality and Size. Nano Lett. 2015, 15, 4985-4992.

(20) Achtstein, A. W.; Prudnikau, A. V.; Ermolenko, M. V.; Gurinovich, L. I.; Gaponenko, S. V.; Woggon, U.; Baranov, A. V.; Leonov, M. Y.; Rukhlenko, I. D.; Fedorov, A. V.; et al. Electroabsorption by 0D, 1D and 2D Nanocrystals: A Comparative Study of CdSe Colloidal Quantum Dots, Nanorods and Nanoplatelets. ACS Nano 2014, 8, 7678-7686.

(21) Bullen, C.; Mulvaney, P. The Effects of Chemisorption on the Luminescence of CdSe Quantum Dots. Langmuir 2006, 22, 30073013.

(22) Brutchey, R.; Hens, Z.; Kovalenko, M. V. Surface Chemistry of Colloidal Semiconductor Nanocrystals: Organic, Inorganic, and Hybrid. In Chemistry of Organo-Hybrids; John Wiley \& Sons, Inc.: Hoboken, NJ, 2015; pp 233-271.

(23) Morris-Cohen, A. J.; Malicki, M.; Peterson, M. D.; Slavin, J. W. J.; Weiss, E. A. Chemical, Structural, and Quantitative Analysis of the Ligand Shells of Colloidal Quantum Dots. Chem. Mater. 2013, 25, $1155-1165$.

(24) Nag, A.; Kovalenko, M. V.; Lee, J.; Liu, W.; Spokoyny, B.; Talapin, D. V. Metal-Free Inorganic Ligands for Colloidal Nanocrystals: $\mathrm{S}^{2-}, \mathrm{H}^{\mathrm{S}-}, \mathrm{Se}^{2-}, \mathrm{HSe}^{-}, \mathrm{Te}^{2-}, \mathrm{HTe}^{-}, \mathrm{TeS}_{3}{ }^{2-}, \mathrm{OH}^{-}$, and $\mathrm{NH}_{2}{ }^{-}$as Surface Ligands. J. Am. Chem. Soc. 2011, 133, 10612-10620.

(25) Kovalenko, M. V.; Manna, L.; Cabot, A.; Hens, Z.; Talapin, D. V.; Kagan, C. R.; Klimov, V. I.; Rogach, A. L.; Reiss, P.; Milliron, D. J.; et al. Prospects of Nanoscience with Nanocrystals. ACS Nano 2015, 9, 1012-1057.

(26) Boles, M. A.; Ling, D.; Hyeon, T.; Talapin, D. V. The Surface Science of Nanocrystals. Nat. Mater. 2016, 15, 141-153.

(27) Abécassis, B.; Tessier, M. D.; Davidson, P.; Dubertret, B. SelfAssembly of CdSe Nanoplatelets into Giant Micrometer-Scale Needles Emitting Polarized Light. Nano Lett. 2014, 14, 710-715.

(28) Guzelturk, B.; Erdem, O.; Olutas, M.; Kelestemur, Y.; Demir, H. V. Stacking in Colloidal Nanoplatelets: Tuning Excitonic Properties. ACS Nano 2014, 8, 12524-12533.

(29) Rowland, C. E.; Fedin, I.; Zhang, H.; Gray, S. K.; Govorov, A. O.; Talapin, D. V.; Schaller, R. D. Picosecond Energy Transfer and Multiexciton Transfer Outpaces Auger Recombination in Binary CdSe Nanoplatelet Solids. Nat. Mater. 2015, 14, 484-489.

(30) Brouwer, A. M. Standards for Photoluminescence Quantum Yield Measurements in Solution (IUPAC Technical Report). Pure Appl. Chem. 2011, 83, 2213-2228.

(31) Grabolle, M.; Spieles, M.; Lesnyak, V.; Gaponik, N.; Eychmüller, A.; Resch-Genger, U. Determination of the Fluorescence Quantum
Yield of Quantum Dots: Suitable Procedures and Achievable Uncertainties. Anal. Chem. 2009, 81, 6285-6294.

(32) Chen, D.; Gao, Y.; Chen, Y.; Ren, Y.; Peng, X. Structure Identification of Two-Dimensional Colloidal Semiconductor Nanocrystals with Atomic Flat Basal Planes. Nano Lett. 2015, 15, 44774482.

(33) Anderson, N. C.; Hendricks, M. P.; Choi, J. J.; Owen, J. S. Ligand Exchange and the Stoichiometry of Metal Chalcogenide Nanocrystals: Spectroscopic Observation of Facile Metal-Carboxylate Displacement and Binding. J. Am. Chem. Soc. 2013, 135, 1853618548.

(34) Kanicky, J. R.; Shah, D. O. Effect of Degree, Type, and Position of Unsaturation on the $\mathrm{pK}_{\mathrm{a}}$ of Long-Chain Fatty Acids. J. Colloid Interface Sci. 2002, 256, 201-207.

(35) Zherebetskyy, D.; Scheele, M.; Zhang, Y.; Bronstein, N.; Thompson, C.; Britt, D.; Salmeron, M.; Alivisatos, P.; Wang, L.-W. Hydroxylation of the Surface of $\mathrm{PbS}$ Nanocrystals Passivated with Oleic Acid. Science 2014, 344, 1380-1384.

(36) Jana, S.; Phan, T. N. T.; Bouet, C.; Tessier, M. D.; Davidson, P.; Dubertret, B.; Abécassis, B. Stacking and Colloidal Stability of CdSe Nanoplatelets. Langmuir 2015, 31, 10532-10539.

(37) Fritzinger, B.; Capek, R. K.; Lambert, K.; Martins, J. C.; Hens, Z. Utilizing Self-Exchange to Address the Binding of Carboxylic Acid Ligands to CdSe Quantum Dots. J. Am. Chem. Soc. 2010, 132, 1019510201.

(38) Owen, J. S.; Park, J.; Trudeau, P.-E.; Alivisatos, A. P. Reaction Chemistry and Ligand Exchange at Cadmium-Selenide Nanocrystal Surfaces. J. Am. Chem. Soc. 2008, 130, 12279-12281.

(39) Gomes, R.; Hassinen, A.; Szczygiel, A.; Zhao, Q.; Vantomme, A.; Martins, J. C.; Hens, Z. Binding of Phosphonic Acids to CdSe Quantum Dots: A Solution NMR Study. J. Phys. Chem. Lett. 2011, 2, 145-152.

(40) Baranov, D.; Fiore, A.; Van Huis, M.; Giannini, C.; Falqui, A.; Lafont, U.; Zandbergen, H.; Zanella, M.; Cingolani, R.; Manna, L. Assembly of Colloidal Semiconductor Nanorods in Solution by Depletion Attraction. Nano Lett. 2010, 10, 743-749.

(41) Schapotschnikow, P.; Hommersom, B.; Vlugt, T. J. H. Adsorption and Binding of Ligands to CdSe Nanocrystals. J. Phys. Chem. C 2009, 113, 12690-12698.

(42) Klinke, C.; Afzali, A.; Avouris, P. Interaction of Solid Organic Acids with Carbon Nanotube Field Effect Transistors. Chem. Phys. Lett. 2006, 430, 75-79.

(43) Langmuir-Blodgett Films; Roberts, G., Ed.; Springer US: New York, 1990.

(44) Rajam, S.; Heywood, B. R.; Walker, J. B. A.; Mann, S.; Davey, R. J.; Birchall, J. D. Oriented Crystallization of $\mathrm{CaCO}_{3}$ under Compressed Monolayers. Part 1. - Morphological Studies of Mature Crystals. J. Chem. Soc., Faraday Trans. 1991, 87, 727-734.

(45) Ugur, I.; Marion, A.; Parant, S.; Jensen, J. H.; Monard, G. Rationalization of the $\mathrm{pK}_{\mathrm{a}}$ Values of Alcohols and Thiols Using Atomic Charge Descriptors and Its Application to the Prediction of Aminoacid pK 's. J. Chem. Inf. Model. 2014, 54, 2200-2213.

(46) Dopico, A. M., Ed.Methods in Membrane Lipids; Methods in Molecular Biology series; Humana Press: Totowa, NJ, 2007.

(47) Lin-Vien, D.; Colthup, N. B.; Fateley, W. G.; Grasselli, J. G. The Handbook of Infrared and Raman Characteristic Frequencies of Organic Molecules; Academic Press: San Diego, 1991.

(48) Nakamoto, K. Infrared and Raman Spectra of Inorganic and Coordination Compounds; Wiley-Interscience: New York, 1978.

(49) Zeleňák, V.; Vargová, Z.; Györyová, K. Correlation of Infrared Spectra of Zinc(II) Carboxylates with Their Structures. Spectrochim. Acta, Part A 2007, 66, 262-272.

(50) Shpaisman, H.; Salomon, E.; Nesher, G.; Vilan, A.; Cohen, H.; Kahn, A.; Cahen, D. Electrical Transport and Photoemission Experiments of Alkylphosphonate Monolayers on GaAs. J. Phys. Chem. C 2009, 113, 3313-3321.

(51) Levine, I.; Weber, S. M.; Feldman, Y.; Bendikov, T.; Cohen, H.; Cahen, D.; Vilan, A. Molecular Length, Monolayer Density, and 
Charge Transport: Lessons from Al-AlOx/alkyl-phosphonate/Hg Junctions. Langmuir 2012, 28, 404-415.

(52) Hostetler, M. J.; Stokes, J. J.; Murray, R. W. Infrared Spectroscopy of Three-Dimensional Self-Assembled Monolayers: nAlkanethiolate Monolayers on Gold Cluster Compounds. Langmuir 1996, 12, 3604-3612.

(53) Meulenberg, R. W.; Bryan, S.; Yun, C. S.; Strouse, G. F. Effects of Alkylamine Chain Length on the Thermal Behavior of CdSe Quantum Dot Glassy Films. J. Phys. Chem. B 2002, 106, 7774-7780.

(54) Hostetler, M. J.; Wingate, J. E.; Zhong, C.-J.; Harris, J. E.; Vachet, R. W.; Clark, M. R; Londono, J. D.; Green, S. J.; Stokes, J. J.; Wignall, G. D.; et al. Alkanethiolate Gold Cluster Molecules with Core Diameters from 1.5 to $5.2 \mathrm{~nm}$ : Core and Monolayer Properties as a Function of Core Size. Langmuir 1998, 14, 17-30.

(55) Boles, M. A.; Talapin, D. V. Self-Assembly of Tetrahedral CdSe Nanocrystals: Effective "Patchiness" via Anisotropic Steric Interaction. J. Am. Chem. Soc. 2014, 136, 5868-5871.

(56) Schapotschnikow, P.; Pool, R; Vlugt, T. J. H. Molecular Simulations of Interacting Nanocrystals. Nano Lett. 2008, 8, 29302934.

(57) Salem, L. Attractive Forces between Long Saturated Chains at Short Distances. J. Chem. Phys. 1962, 37, 2100-2113.

(58) Pellegrino, T.; Manna, L.; Kudera, S.; Liedl, T.; Koktysh, D.; Rogach, A. L.; Keller, S.; Rädler, J.; Natile, G.; Parak, W. J. Hydrophobic Nanocrystals Coated with an Amphiphilic Polymer Shell: A General Route to Water Soluble Nanocrystals. Nano Lett. 2004, 4, 703-707.

(59) Wu, X.; Liu, H.; Liu, J.; Haley, K. N.; Treadway, J. A.; Larson, J. P.; Ge, N.; Peale, F.; Bruchez, M. P. Immunofluorescent Labeling of Cancer Marker Her2 and Other Cellular Targets with Semiconductor Quantum Dots. Nat. Biotechnol. 2002, 21, 41-46.

(60) Yu, W. W.; Chang, E.; Falkner, J. C.; Zhang, J.; Al-Somali, A. M.; Sayes, C. M.; Johns, J.; Drezek, R.; Colvin, V. L. Forming Biocompatible and Nonaggregated Nanocrystals in Water Using Amphiphilic Polymers. J. Am. Chem. Soc. 2007, 129, 2871-2879.

(61) Zhang, F.; Lees, E.; Amin, F.; Rivera-Gil, P.; Yang, F.; Mulvaney, P.; Parak, W. J. Polymer-Coated Nanoparticles: A Universal Tool for Biolabelling Experiments. Small 2011, 7, 3113-3127.

(62) Palui, G.; Aldeek, F.; Wang, W.; Mattoussi, H. Strategies for Interfacing Inorganic Nanocrystals with Biological Systems Based on Polymer-Coating. Chem. Soc. Rev. 2015, 44, 193-227.

(63) Katari, J. E. B.; Colvin, V. L.; Alivisatos, A. P. X-Ray Photoelectron Spectroscopy of CdSe Nanocrystals with Applications to Studies of the Nanocrystal Surface. J. Phys. Chem. 1994, 98, 41094117.

(64) Hadar, I.; Abir, T.; Halivni, S.; Faust, A.; Banin, U. SizeDependent Ligand Layer Dynamics in Semiconductor Nanocrystals Probed by Anisotropy Measurements. Angew. Chem., Int. Ed. 2015, 54, 12463-12467.

(65) Walker, D. A.; Leitsch, E. K.; Nap, R. J.; Szleifer, I.; Grzybowski, B. A. Geometric Curvature Controls the Chemical Patchiness and SelfAssembly of Nanoparticles. Nat. Nanotechnol. 2013, 8, 676-681.

(66) Jones, M. R.; Macfarlane, R. J.; Prigodich, A. E.; Patel, P. C.; Mirkin, C. A. Nanoparticle Shape Anisotropy Dictates the Collective Behavior of Surface-Bound Ligands. J. Am. Chem. Soc. 2011, 133, 18865-18869.

(67) McNeill, V. F.; Wolfe, G. M.; Thornton, J. A. The Oxidation of Oleate in Submicron Aqueous Salt Aerosols: Evidence of a Surface Process. J. Phys. Chem. A 2007, 111, 1073-1083.

(68) Wuelfing, W. P.; Gross, S. M.; Miles, D. T.; Murray, R. W. Nanometer Gold Clusters Protected by Surface-Bound Monolayers of Thiolated Poly(ethylene Glycol) Polymer Electrolyte. J. Am. Chem. Soc. 1998, 120, 12696-12697.

(69) Woehrle, G. H.; Brown, L. O.; Hutchison, J. E. ThiolFunctionalized, 1.5-nm Gold Nanoparticles through Ligand Exchange Reactions: Scope and Mechanism of Ligand Exchange. J. Am. Chem. Soc. 2005, 127, 2172-2183.
(70) Asakura, S.; Oosawa, F. On Interaction between Two Bodies Immersed in a Solution of Macromolecules. J. Chem. Phys. 1954, 22, $1255-1256$.

(71) Goff, M. J.; Suppes, G. J.; Dasari, M. A. Interpreting Freezing Point Depression of Stearic Acid and Methyl Stearate. Fluid Phase Equilib. 2005, 238, 149-156.

(72) Kosolapoff, G. M. Chemistry of Aliphatic Phosphonic Acids. II. Dielectric Constants and Viscosity of Some Higher Alkylphosphonates. J. Am. Chem. Soc. 1954, 76, 615-617.

(73) Campbell, I.; Rubin, S.; Zawodzinski, T.; Kress, J.; Martin, R.; Smith, D.; Barashkov, N.; Ferraris, J. Controlling Schottky Energy Barriers in Organic Electronic Devices Using Self-Assembled Monolayers. Phys. Rev. B: Condens. Matter Mater. Phys. 1996, 54, R14321-R14324.

(74) Brown, P. R.; Kim, D.; Lunt, R. R.; Zhao, N.; Bawendi, M. G.; Grossman, J. C.; Bulović, V. Energy Level Modification in Lead Sulfide Quantum Dot Thin Films through Ligand Exchange. ACS Nano 2014, $8,5863-5872$. 